Short communication

\title{
A novel control condition for spatial learning in the Morris water maze
}

\author{
Daniel N. Barry*, Sean Commins \\ Department of Psychology, Maynooth University, Co. Kildare, Ireland
}

\section{A R T I C L E I N F O}

\section{Keywords:}

Morris water maze

Spatial learning

Control condition

\begin{abstract}
A B S T R A C T
Background: Assessing markers of neural activity in rodent behavioural tasks benefits from meaningful comparison with matched control conditions. The Morris water maze is a widely used learning and memory task, but currently implemented control conditions are not optimal.

New method: We created a novel control condition involving random escape platform placement for each trial, while increasing the frequency of platforms throughout the training period. These control rats could therefore escape the maze by swimming in a random fashion and were not required to learn a platform location. Crucially, because the number of available escape platforms increased throughout training, their escape latencies should decline in line with rats who were learning a fixed platform location.

Results: No evidence of place learning was observed in this novel condition, but their swimming behaviour, including escape latency, distance and velocity were well matched to spatially-trained rats throughout training. Further, they did not display stress-related behaviour.

Comparison with existing methods: We contrasted this new control condition against a frequently used control where animals swim freely in the maze and showed behaviour was more closely matched to spatially-trained animals.

Conclusions: This novel control condition represents a significant advance from those currently available and may assist in the interpretation of task-related neural activity.
\end{abstract}

\section{Introduction}

The Morris water maze task is widely used to study spatial navigation and memory in rodents (Morris, 1981). Animals are placed into a pool of water and must use environmental cues to locate a submerged platform. The procedure can be adapted to investigate egocentric navigational strategies such as path integration, through training in darkness (Commins et al., 1999), taxic strategies involving the use of cues proximal to the platform, or spatial working memory, where the escape platform is relocated on each day of training (Paul et al., 2009). It is also commonly used as a sensitive test of spatial reference memory, where the platform remains fixed across multiple days of training. However, the water maze task can be problematic when assessing and interpreting corresponding neural activity as the stress of swimming can lead to an increase in commonly used immediate early gene markers such as c-Fos, Zif268, Arc, and metabolic measures such as uptake of 2-deoxyglucose (Cullinan et al., 1995; Duncan et al., 1993; Ons et al., 2004). Therefore, having a behaviourally matched control condition is desirable (Johnson and Besselsen, 2002). One common control condition involves animals swimming freely in the maze without an escape platform present, however this results in comparable hippocampal activation to rats performing a spatial working memory task, and even stronger activation of the lateral entorhinal cortex, perirhinal cortex and dorsal subiculum in the free-swimming group (Shires and Aggleton, 2008), suggesting even brief periods of forced swimming result in heightened stress. One alternative which has been proposed is to train animals to swim around the maze at a fixed distance from the pool wall (Shires and Aggleton, 2008). While animals do not learn a particular spatial location, they must still adopt a particular navigational strategy which requires extended training. Here, we created a novel control condition which encouraged a random swimming pattern, but preserved the experience of escaping the maze, by changing the escape platform location for every trial. Therefore, rats could escape the maze without learning a platform location. To match swimming times with rats who were learning a fixed platform location and getting faster at escaping the maze with further training, we increased the number of randomly placed platforms each day. We compared the swimming behaviour of this condition to spatially-trained rats, as well as a freeswimming condition and a further control condition where a single platform changed location during each trial.

\footnotetext{
* Corresponding author. Present address: Wellcome Centre for Human Neuroimaging, 12 Queen Square, London WC1N 3AR, United Kingdom.

E-mail address: daniel.barry@ucl.ac.uk (D.N. Barry).
} 


\section{Materials and methods}

\subsection{Subjects}

Male Wistar rats ( $n=28$; three months old, 200-300 g) were used as subjects (obtained from Charles River Laboratories, UK). Animals were housed three per cage, in a temperature-controlled environment $\left(21 \pm 1{ }^{\circ} \mathrm{C}\right.$ ), which was maintained on a fixed 12:12 h light-dark cycle (07:00-19:00). All rats were given ad libitum access to food and water. Experimentation took place during the light phase and all subjects were well handled before experimentation began. The rats had no prior exposure to the maze and were experimentally naïve. Rats were randomly allocated to one of four groups ( $n=7$ per group). Power calculations to determine the appropriate sample size were performed using $\mathrm{G}^{*}$ Power (http://www.gpower.hhu.de/). As we were using a repeated-measures ANOVA with within-between interaction, across four groups and five days of training, with a specified high power of 0.9 and moderate effect size of 0.3 , we calculated an overall $\mathrm{n}$ of 28 , with seven per group. In addition, previous behavioural investigations in our lab using the same water maze apparatus and training protocol have used equivalent sample sizes (Commins et al., 2003; Harvey et al., 2009; Kealy et al., 2008).

\subsection{Apparatus}

The water maze consisted of a black, circular fibreglass pool (diameter of $170 \mathrm{~cm}$, depth of $36 \mathrm{~cm}$ ), elevated $70 \mathrm{~cm}$ above the floor (Harvey et al., 2008). The maze was filled with water to a depth of $20 \mathrm{~cm}$ and maintained at $20 \pm 1{ }^{\circ} \mathrm{C}$. The escape platform $(18 \mathrm{~cm}$ height; $13.5 \mathrm{~cm}$ diameter) was submerged $2 \mathrm{~cm}$ below the water surface. The water maze area was enclosed by a black curtain with three cues available, two $25 \mathrm{~W}$ bulbs which were suspended from the ceiling in the northeast and southeast quadrants, at $75 \mathrm{~cm}$ from the edge of the pool and at an angle of $60^{\circ}$, and a rectangular piece of white card ( $55 \mathrm{~cm} \mathrm{x} 81 \mathrm{~cm}$ ) which was also suspended from the ceiling against the black background, on the west side of the maze (Fig. 1).

\subsection{Procedure}

The spatially-trained group ( $\mathrm{n}=7$ ) was trained for five consecutive days in the water maze, with four trials per day. Animals were placed into the water maze from one of four starting positions, either north, south, east or west, with each starting position used just once per day. Animals were allowed $60 \mathrm{~s}$ to locate the escape platform, after which they would be guided to the platform by the experimenter and allowed to remain there for $15 \mathrm{~s}$. Following an inter-trial interval of $10 \mathrm{~s}$, the animal was placed back into the maze to begin the next trial (Fig. 1A). Rats in a "multiple variable platform" group $(n=7)$ were also trained for four trials per day for five days but were tasked with locating an escape platform which could be in one of 20 possible locations which changed on every trial (Fig. 1B). The number of available escape platforms in this condition increased by one per day, from one platform on day one to five on day five. A platform location was never repeated on the same day. A "single variable platform" group $(n=7)$ followed a similar training protocol but only had one randomly-placed escape platform available for every trial over the five days (Fig. 1C). A freeswimming group ( $n=7$ ) swam in the maze in the absence of an escape platform for the same amount of time as the spatially-trained animals (Fig. 1D). A camera was positioned directly above the centre of the maze which recorded the animal's movements for each experimental trial. This information was collected by the digital tracking software EthoVision (Noldus Information Technologies, Wageningen, Netherlands), where an analysis of escape latencies, distance travelled, velocity and areas searched for each animal on every trial was calculated.

\subsection{Data analysis}

Escape latencies, distance travelled and velocity for each trial were calculated, and averaged to produce a mean for every animal for each day. To provide a general measure of the successful learning and retention of a specific platform location (spatial reference memory), we calculated the time spent searching in each quadrant on day five of training. This represented a percentage of total escape latency; the proportion of time spent searching in each quadrant until a platform
A
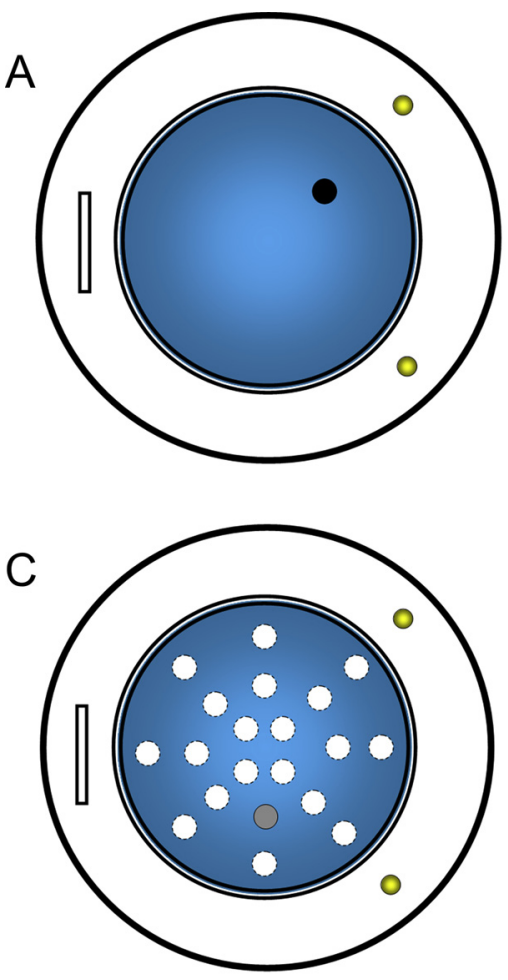
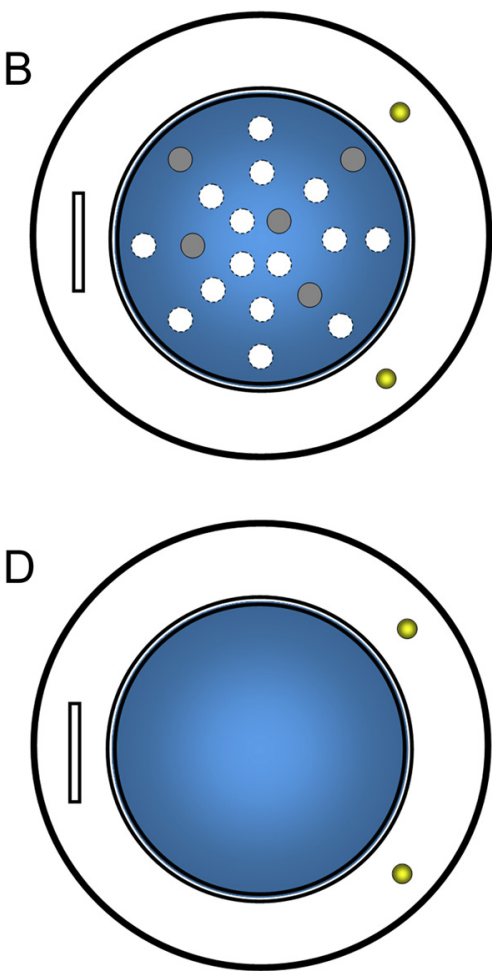

Fig. 1. Morris water maze platform configurations. (A) Fixed platform in the spatially-trained group. (B) Multiple variable platform group possible escape locations (white) with sample locations for one trial on day five (grey). (C) Single variable platform group possible escape locations (white) with sample location for one trial on day five (grey). (D) Free-swimming group configuration with no escape platform present. 
was discovered. In a similar analysis to detect if control animals were temporarily encoding and retrieving the location of changing platform locations (spatial working memory), we assessed percentage time searching more specifically around the previous trial's escape platform position on each day, in an area comprising $5 \%$ of the total maze swimming area. Again, this time represented the percentage of total escape latency on each trial. To quantify thigmotactic behaviour which may be indicative of stress (Treit and Fundytus, 1988) percentage time spent swimming in corridor of $16 \mathrm{~cm}$ width around the circumference of the swimming area was analysed. Escape latencies, distance travelled and velocity were analysed using mixed between-within analyses of variance (ANOVA). For parsimony, we selectively report interaction effects between day and condition over main effects, if present. Subsequent one-way ANOVAs with Tukey post-hoc tests were then performed on days one and five to assess group differences at the beginning and end of training. To compare time spent in water maze quadrants for each group on day five, one-way repeated measures ANOVAs were performed, with Bonferroni-corrected comparisons. To compare time spent in a previous platform location across groups, one-way ANOVAs were performed, with Tukey post-hoc tests. All ANOVAs were subjected to Greenhouse-Geisser adjustment to the degrees of freedom if Mauchly's sphericity test identified that sphericity had been violated. All statistical analyses were carried out using IBM SPSS Statistics for Windows, version 20 (IBM Corp., Armonk, N.Y., USA).

\subsection{Ethical considerations}

Guidelines for the maintenance and experimentation of animals conformed to the Department of Health and Children (Ireland) guidelines under statutory instrument (S.I.) No. 543 of 2012 and the European directive 2010/63/EU. The Maynooth University ethics committee also approved all experimental work.

\section{Results}

\subsection{Escape latency}

As the free-swimming group were matched to the spatially-trained group for time spent in the maze, they were excluded from the escape latency analysis. A 3 (condition) x 5 (day) mixed factorial ANOVA revealed a significant interaction effect between day and group $\left(F_{(8,72)}=3.53, p=0.002\right)$, with no difference between groups on the first day of training $\left.F_{(2,18)}=0.91, p=0.913\right)$, as opposed to day five $\left(F_{(2,18)}=16.82, p<0.001\right)$, when the spatially-trained $(p<0.001)$ and multiple variable group $(p<0.001)$ spent less time in the maze than the single variable platform group (Fig. 2A). The multiple variable platform group and spatially-trained group therefore spent an equivalent amount of time in the maze, which was not the case when only one randomly placed escape platform was present.

\subsection{Distance travelled}

A 4 (condition) x 5 (day) mixed factorial ANOVA revealed an interaction between day and group for distance travelled $\left(F_{(12,96)}=20.34, p=0.004\right)$, with the groups swimming a comparable distance in the maze on day one $\left(F_{(3,24)}=0.39, p=0.758\right)$, but not on day five $\left(F_{(3,24)}=6.97, p=0.002\right.$; Fig. $\left.2 \mathrm{~B}\right)$, when the distance travelled was higher in the single variable platform group than the spatially-trained $(p=0.011)$, multiple variable platform $(p=0.001)$ and free-swimming group ( $p=0.037)$. The multiple variable platform group and the spatially-trained group were therefore also well matched on distance travelled.

\subsection{Velocity}

A 4 (condition) x 5 (day) mixed factorial ANOVA showed an interaction effect between day and group for swimming velocity, $\left(F_{(8.69,69.51)}=3.71, p<0.001\right.$; Fig. $\left.2 \mathrm{C}\right)$. Subsequent one-way ANOVAs showed there was no difference between the groups on day one, $\left(F_{(3,24)}=2.15, p=0.120\right)$, but on day five a significant difference between the groups emerged, $\left(F_{(3,24)}=3.46, p=0.032\right)$, where velocity of the free-swimming group was significantly higher than the multiple variable platform group ( $p=0.041)$. As with escape latency and distance travelled, swimming speed did not differ between the novel multiple variable platform group and the spatially-trained group.

\subsection{Thigmotaxis}

A 4 (condition) x 5 (day) mixed factorial ANOVA revealed a significant interaction between day and group on a measure of thigmotactic behaviour $\left(F_{(12,96)}=2.02, p=0.030\right.$; Fig. $\left.2 \mathrm{D}\right)$. Subsequent oneway ANOVAs confirmed a significant difference between the groups on day one, $\left(F_{(3,24)}=3.83, p=0.022\right)$, where the free-swimming group displayed more thigmotactic behaviour than the spatially-trained ( $p=$ $0.043)$, multiple variable platform $(p=0.049)$, with the single variable platform group just shy of significance $(p=0.056)$. This group difference was still evident on day five $\left(F_{(3,24)}=11.41, p<0.001\right)$, where the free-swimming group spent more time swimming around the edge of the pool than the spatially-trained $(p<0.001)$, multiple variable platform $(p<0.001)$ and single variable platform group $(p=0.004)$. The multiple variable platform group therefore did not appear disproportionately stressed in comparison with spatially-trained rats, unlike the free-swimming condition.

\subsection{Quadrant analysis}

On day five, a significant difference in quadrant search time was observed in the spatially-trained group, $\left(F_{(3,18)}=14.64, p<0.001\right)$, where more time was spent searching in the northeast quadrant than the southeast $(p=0.008)$ and southwest $(p=0.008$; Fig. $2 \mathrm{E})$. A significant effect for quadrant was not found in the multiple variable platform group $\left(F_{(3,18)}=0.89, p=0.468\right)$, single variable platform group $\left(F_{(3,18)}=0.29, p=0.830\right)$, or the free-swimming group $\left(F_{(3,18)}=0.34, p=0.797\right)$. Therefore only the reference memory group showed evidence of the learning and retention of a specific platform location, whereas the novel multiple platform group did not develop a preference for searching in any area of the maze as training progressed.

\subsection{Searching in previous trial platform area}

On day one, there was a difference in previous platform area search time between conditions $\left(F_{(2,18)}=8.77, p=0.002\right)$, where the spatially-trained group returned to the platform area located on the previous trial more often than the multiple variable $(p=0.011)$ and single variable platform group ( $p=0.003$; Fig. $2 \mathrm{~F}$ ). A similar pattern was found on day two $\left(F_{(2,18)}=8.64, p=0.002\right)$, with the spatially-trained group searching more in the previous platform area than the multiple variable $(p=0.009)$ and single variable platform group $(p=0.004)$. A significant effect for condition was again found on day three $\left(F_{(2,18)}=\right.$ $3.92, p=0.039)$, however post-hoc tests between the spatially-trained group and the multiple variable platform group $(p=0.052)$ and the single variable platform group $(p=0.081)$ did not reach statistical significance. On day four, a significant difference between the groups was again found $\left(F_{(2,18)}=15.39, p<0.001\right)$, with the spatiallytrained group again spending a significantly higher percentage of time searching in the previous platform area than the multiple variable platform group $(p<0.001)$ and the single variable platform group $(p=0.003)$. On the final day of training, a main effect was found for group $\left(F_{(2,18)}=15.44, p<0.001\right)$, the spatially-trained group again spending more time in the previous platform area than the multiple variable platform group $(p<0.001)$, and the single variable platform 

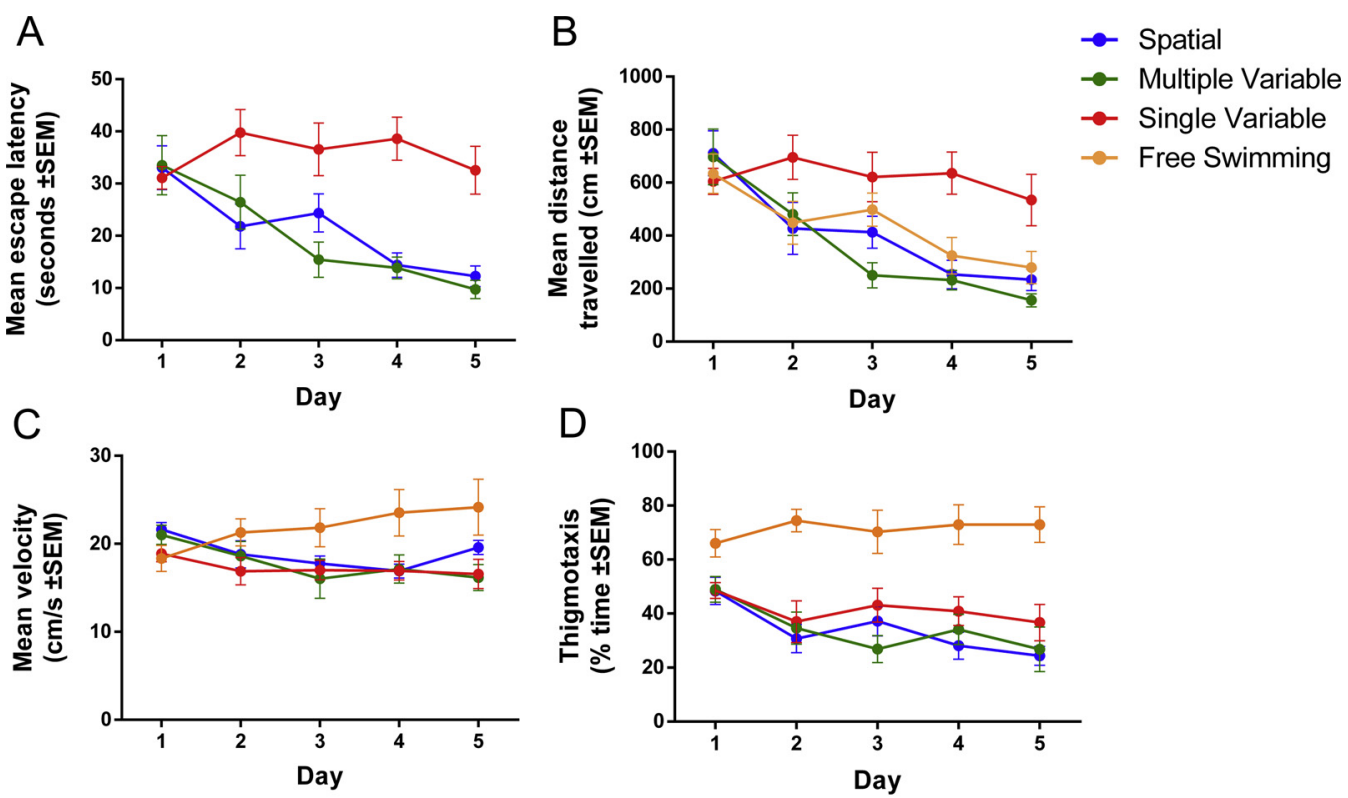

$E$
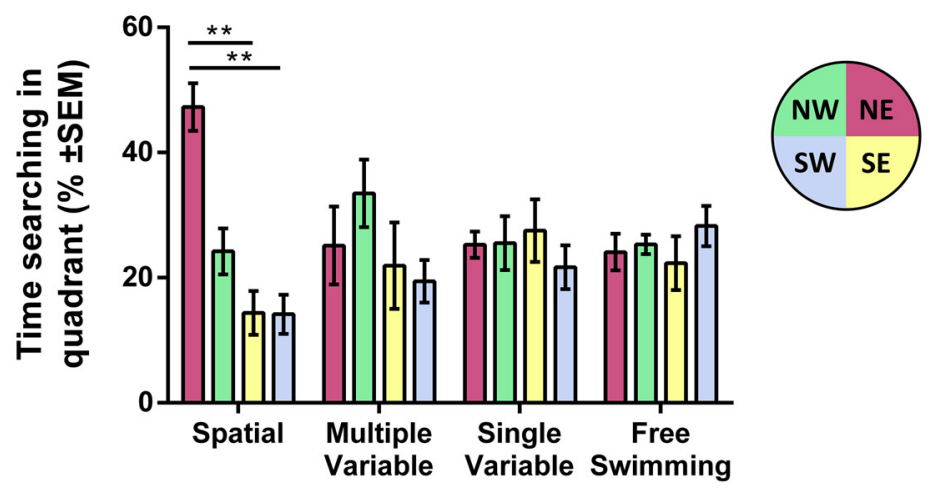

$\mathrm{F}$

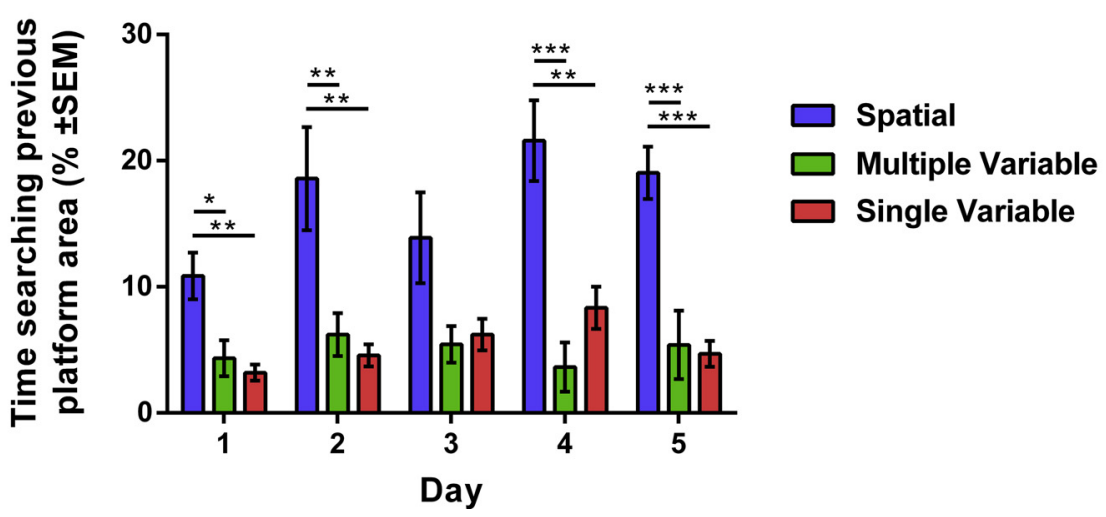

Fig. 2. (A) Escape latencies for spatially-trained, multiple variable platform and single variable platform conditions over five days of training. (B) Distance travelled for all groups. (C) Swimming velocity for all groups. (D) Thigmotactic behaviour for all groups. (E) Preference for a maze quadrant on day five of training. (F). Percentage time searching in platform location of previous trial across five days of training. ${ }^{*} \mathrm{p}<0.05,{ }^{* *} \mathrm{p}<0.01,{ }^{* * *} \mathrm{p}<0.001$.

group ( $p<0.001)$. This suggests while the spatially-trained group kept the previous platform location in memory within and across days, the novel multiple platform group did not retain this information as they were no more likely to search the location where they had just located an escape platform than would be expected by chance.

\section{Discussion}

Our novel control condition for the Morris water maze involved varying the escape platform location on every trial and adding additional platforms as training progressed. This condition controlled for many aspects of water maze training which may influence neural activation, including immersion in water, duration of swim time, swimming speed, exposure to cues, the experience of escaping the maze, time spent on the platform, handling by the experimenter, and stress as measured by thigmotactic behaviour. The resulting benefit is that the only difference between the reference memory condition and this control condition, is that the former learn and retain a specific platform location, and the latter show no evidence of either working or reference memory. This procedure could also be adapted to serve as a 
control for other water maze tasks, such as those relying on egocentric strategies or working memory.

This novel control condition stands to improve knowledge about brain activity, as future studies assessing neural differences between this condition and a spatial memory condition can more confidently ascribe such changes to learning than extraneous factors in this task such as exercise or stress. Such neural changes may include markers of plasticity (Guzowski et al., 2001), neurogenesis (Ambrogini et al., 2000), dendritic spine density (O'Malley et al., 2000), and post-training enhancements in long-term potentiation (Schulz and Korz, 2010).

This novel condition affords numerous behavioural advantages in comparison with presently used control conditions. Control animals kept in their home cage are not exposed at all to the water maze, and therefore are of limited utility when interpreting brain activation (Shires and Aggleton, 2008). Commonly used "free swimming" controls rely on the experimenter to rescue them from the maze, and accordingly experience increased stress relative to animals who can escape the maze of their own volition (Kavushansky et al., 2006). Animals can learn to escape the water maze by swimming towards a visible randomly placed platform and therefore not relying on environmental cues. However this is an unsatisfactory control as swim paths are invariably shorter relative to navigation towards an invisible platform (Lopez et al., 2012). An alternative potential condition which does not rely on distal cues, is the random placement of an invisible platform, but at a fixed distance from a local visible beacon (Pearce et al., 1998). However animals rapidly adopt this strategy over the first few sessions, making it difficult to match to a reference memory condition. A related control condition is to train rats to swim at a fixed distance from the pool wall until a randomly-placed platform is located within this circular corridor (Shires and Aggleton, 2008). However encouraging animals to adopt such a strategy requires extensive training. The control condition devised here does not suffer from any of these limitations, with behaviour well-matched throughout training, and consequently we feel the method represents a valuable tool for researchers to adequately control their experiments. One potential modification to our condition rather than relocating platforms is to randomly rotate the environmental cues after each trial. However we believe this would be less effective because animals could default to the use of vestibular information to return to the fixed position of a previously located platform (McGauran et al., 2005).

A navigational strategy cannot be completely excluded in this novel control condition, such as the use of distal cues to systematically explore the maze. However escape latencies in the single variable platform group did not decrease over training suggesting such a strategy was not developed over time. Some incidental spatial learning is possible in this novel control condition, however encoding up to 20 different locations over training when this information is not useful for task performance would seem unlikely. The unpredictable nature of escape platform locations in this group could arguably be more stressful than a reference memory task. However, we did not observe any behavioural differences between the two groups. In summary, this novel control condition represents a significant advancement over currently deployed control conditions in the Morris water maze and is likely to be useful for meaningful interpretation of neural markers of plasticity during learning and retrieval.

\section{Declaration of interests}

None.

\section{Funding sources}

This work was funded by an Irish Research Council scholarship and an NUIM John and Pat Hume scholarship awarded to D.N.B. The funding bodies did not have a role in study design, data collection or analysis.

\section{References}

Ambrogini, P., Cuppini, R., Cuppini, C., Ciaroni, S., Cecchini, T., Ferri, P., et al., 2000. Spatial learning affects immature granule cell survival in adult rat dentate gyrus. Neurosci. Lett. 286 (1), 21-24.

Commins, S., Gemmell, C., Anderson, M., Gigg, J., O’Mara, S.M., 1999. Disorientation combined with bilateral parietal cortex lesions causes path integration deficits in the water maze. Behav. Brain Res. 104 (1-2), 197-200. https://doi.org/10.1016/S01664328(99)00094-7.

Commins, S., Cunningham, L., Harvey, D., Walsh, D., 2003. Massed but not spaced training impairs spatial memory. Behav. Brain Res. 139 (1), 215-223. https://doi. org/10.1016/S0166-4328(02)00270-X.

Cullinan, W.E., Herman, J.P., Battaglia, D.F., Akil, H., Watson, S.J., 1995. Pattern and time course of immediate early gene expression in rat brain following acute stress. Neuroscience 64 (2), 477-505. https://doi.org/10.1016/0306-4522(94)00355-9.

Duncan, G.E., Johnson, K.B., Breese, G.R., 1993. Topographic patterns of brain activity in response to swim stress: assessment by 2-deoxyglucose uptake and expression of Foslike immunoreactivity. J. Neurosci. 13 (9), 3932-3943. https://doi.org/10.1523/ JNEUROSCI.13-09-03932.1993.

Guzowski, J.F., Setlow, B., Wagner, E.K., McGaugh, J.L., 2001. Experience-dependent gene expression in the rat hippocampus after spatial learning: a comparison of the immediate-early genes Arc, c-fos, and zif268. J. Neurosci. 21 (14), 5089-5098.

Harvey, D.R., McGauran, A.M., Murphy, J., Burns, L., McMonagle, E., Commins, S., 2008. Emergence of an egocentric cue guiding and allocentric inferring strategy that mirrors hippocampal brain-derived neurotrophic factor (BDNF) expression in the Morris water maze. Neurobiol. Learn. Mem. 89 (4), 462-479. https://doi.org/10.1016/j. nlm.2007.08.013.

Harvey, D.R., Brant, L., Commins, S., 2009. Differences in cue-dependent spatial navigation may be revealed by in-depth swimming analysis. Behav. Processes 82 (2), 190-197. https://doi.org/10.1016/j.beproc.2009.06.008.

Johnson, P.D., Besselsen, D.G., 2002. Practical aspects of experimental design in animal research. ILAR J. 43 (4), 202-206. https://doi.org/10.1093/ilar.43.4.202.

Kavushansky, A., Vouimba, R.M., Cohen, H., Richter-Levin, G., 2006. Activity and plasticity in the CA1, the dentate gyrus, and the amygdala following controllable vs. uncontrollable water stress. Hippocampus 16 (1), 35-42. https://doi.org/10.1002/ hipo. 20130.

Kealy, J., Diviney, M., Kehoe, E., McGonagle, V., O'Shea, A., Harvey, D., Commins, S., 2008. The effects of overtraining in the Morris water maze on allocentric and egocentric learning strategies in rats. Behav. Brain Res. 192 (2), 259-263. https://doi. org/10.1016/j.bbr.2008.04.009.

Lopez, J., Herbeaux, K., Cosquer, B., Engeln, M., Muller, C., Lazarus, C., et al., 2012. Context-dependent modulation of hippocampal and cortical recruitment during remote spatial memory retrieval. Hippocampus 22 (4), 827-841. https://doi.org/10. 1002/hipo.20943.

McGauran, A.M., O'Mara, S.M., Commins, S., 2005. Vestibular influence on water maze retention: transient whole body rotations improve the accuracy of the cue-based retention strategy. Behav. Brain Res. 158 (1), 183-187. https://doi.org/10.1016/j. bbr.2004.08.013.

Morris, R.G., 1981. Spatial localization does not require the presence of local cues. Learn. Motiv. 12 (2), 239-260. https://doi.org/10.1016/0023-9690(81)90020-5.

O'Malley, A., O'Connell, C., Murphy, K.J., Regan, C.M., 2000. Transient spine density increases in the mid-molecular layer of hippocampal dentate gyrus accompany consolidation of a spatial learning task in the rodent. Neuroscience 99 (2), 229-232.

Ons, S., Marti, O., Armario, A., 2004. Stress-induced activation of the immediate early gene Arc (activity-regulated cytoskeleton-associated protein) is restricted to telencephalic areas in the rat brain: relationship to c-fos mRNA. J. Neurochem. 89 (5), 1111-1118. https://doi.org/10.1111/j.1471-4159.2004.02396.x.

Paul, C.M., Magda, G., Abel, S., 2009. Spatial memory: theoretical basis and comparative review on experimental methods in rodents. Behav. Brain Res. 203 (2), 151-164. https://doi.org/10.1016/j.bbr.2009.05.022.

Pearce, J.M., Roberts, A.D., Good, M., 1998. Hippocampal lesions disrupt navigation based on cognitive maps but not heading vectors. Nature 396 (6706), 75-77. https:// doi.org/10.1038/23941.

Schulz, K., Korz, V., 2010. Emotional and cognitive information processing: relations to behavioral performance and hippocampal long-term potentiation in vivo during a spatial water maze training in rats. Learn. Mem. 17 (11), 552-560. https://doi.org/ $10.1101 / \mathrm{lm} .1855610$.

Shires, K.L., Aggleton, J.P., 2008. Mapping immediate-early gene activity in the rat after place learning in a water-maze: the importance of matched control conditions. Eur. J. Neurosci. 28 (5), 982-996. https://doi.org/10.1111/j.1460-9568.2008.06402.x.

Treit, D., Fundytus, M., 1988. Thigmotaxis as a test for anxiolytic activity in rats. Pharmacol. Biochem. Behav. 31 (4), 959-962. https://doi.org/10.1016/00913057(88)90413-3. 